\title{
A VLA Survey for Radio Pulsars in the Galactic Center
}

\author{
T. Joseph W. Lazio \\ Naval Research Laboratory, Code 7213, Washington, DC 20375-5351 \\ USA
}

James M. Cordes

Department of Astronomy, Cornell University and National Astronomy B Ionosphere Center, Ithaca, NY 14853-6801 USA

\begin{abstract}
A radio pulsar in the Galactic center will suffer $350 \nu_{\mathrm{GHz}}^{-4}$ seconds of pulse broadening. An imaging search for compact sources is far less desensitized by angular broadening than a periodicity search is affected by pulse broadening. We have conducted an imaging survey of the GC using the VIA and have detected approximately 200 sources. Six compact, steep-spectrum sources have been identified; additional compact sources with no spectral information have also been identified. Additional observations are in progress.
\end{abstract}

\section{Radio Pulsars in the Galactic Center}

The central $300 \mathrm{pc}\left(2^{\circ}\right.$ at $\left.8.5 \mathrm{kpc}\right)$ of the Galaxy has the highest stellar densities in the Galaxy, with a contral density near $10^{8} \mathrm{M}_{\odot} \mathrm{pc}^{-3}$. This region contains clusters of hot, presumably young, massive stars; large-scale features thought to originate from powerful stellar winds and supernovae; and a concentration of $\mathrm{X}$-ray binaries. The GC should also have a large population of radio pulsars, both recently formed and recycled. Radio pulsars in the GC would provide opportunities to probe its magnetoionic material, gravitational potential, and star formation. However, the pulsar census contains only a handful within a few degrees of the GC, none with distances in (or beyond) the GC.

Extreme pulse broadening causes this deficit of radio pulsars. Compact GC sources ( $\mathrm{Sgr} \mathrm{A}^{*}$ and $\mathrm{OH}$ masers) display enhanced angular broadening, $1^{\prime \prime}$ at $1 \mathrm{GHz}$ (van Langevelde et al. 1992; Frail et al. 1994; Yusef-Zadeh et al. 1994); the implied pulse broadening is $350 \nu_{\mathrm{GH}}^{-4}$ seconds (Cordes \& Lazio 1997).

A periodicity search would require $\nu \geq 10 \mathrm{GHz}$ in order for the pulse broadening to be comparable to typical pulse periods. In constrast, sufficiently luminous compact sources (e.g., Sgr $A^{*}$ and the OH masers) can be imaged at much lower frequencies. Cordes \& Lazio (1997) estimated that the GC contains 1-1000 sufficiently luminous pulsars, depending upon the vigor of recent starbursts.

We have observed the GC with the A-configuration VLA at a frequency of $1.4 \mathrm{GHz}$. The VLA provides a resolution of 1".5, comparable to that expected for highly scattered GC sources. Furthermore, with a field of view of about $15^{\prime}$, the inner $1^{\circ}$ of the CC can covered cfliciently by an overlapping hexagonal grid. 


\section{Radio Pulsar Candidates}

We identify radio pulsar candidates as compact, steep-spectrum objects. Based on the diameters of $\operatorname{Sgr} A^{*}$ and the $\mathrm{OH}$ masers, pulsar diameters should be roughly $1^{\prime \prime}$. Spectral information is obtained from a cross-comparison of our survey with a $5 \mathrm{GHz}$ survey of the GC (Becker et al. 1994).

We have detected (a) 3 sources with steep spectral indices; (b) 3 sources that should have been seen in the $5 \mathrm{GHz}$ survey unless their spectral indices are $\alpha<-1\left(S \propto \nu^{\alpha}\right)$; and (c) 28 sources with the expected diameters for heavily scattered pulsars, but outside the latitude limit, $|b|<0.4$, of the 5 GHz survey.

Additional $5 \mathrm{GHz}$ VLA observations of these pulsar candidates have been obtained. Our objectives are (1) Verify or obtain the spectral index; and (2) Verify that source diameters scale as $\lambda^{2}$, as expected for scattered pulsars. Sources that are both steep spectrum and scattered will be subjects of a periodicity search at a frequency high enough to defeat pulse broadening $(\nu>8$ GHz).

\section{Supernovae in the Galactic Center}

Supernovae may be a source of the $10^{7} \mathrm{~K}$, X-ray gas filling the central $1^{\circ}$ of the $\mathrm{GC}$, and their number provides a constraint on the vigor of recent starbursts. The number of supernovae is related to the number of pulsars (and candidates, Cordes \& Lazio 1997): $N_{\mathrm{psr}}=\int_{v} f_{L} f_{b} N_{\mathrm{SN}}$, where $f_{v}$ is the fraction of pulsars with velocities low enough to remain bound to the $\mathrm{GC}, f_{L}$ is the fraction luminous enough to be detected, and $f_{b}$ is the fraction beamed toward Earth. Cordes $\&$ Lazio (1997) estimated $f_{v} f_{L} f_{b} \sim 10^{-3}-10^{-4}$.

Using our 6 pulsar candidates, we can constrain $N_{\mathrm{SN}}$. We tiake $N_{\mathrm{psr}} \sim$ 10. Clearly the number of pulsars could be smaller; the number could also be larger, though not by more than a factor of a few. We estimate $N_{\mathrm{SN}} \lesssim 10^{4}-10^{5}$ within the past few million years (approximate pulsar lifetime). This number is consistent with the number required to produce the X-ray emitting plasma ( $\sim$

$10^{3}$ ) and number required to produce the $1.8 \mathrm{MeV}$ emission from ${ }^{26} \Lambda \mathrm{I}\left(\sim 10^{5}\right)$.

Acknowledgments. Radio astronomy research at the NRL is supported by the Office of Naval Research.

\section{References}

Becker, R. H., White, R. L., Helfand, D. J., \& /oonematkermani, S. 1994, ApJS, 91,347

Cordes, J. M. \& Lazio, T. J. W. 1997, ApJ, 475, 557

Frail, D. A., Diamond, P. J., Cordes, J. M., \& van Langevelde, I. J. 1994, ApJ, $427, \mathrm{~L} 43$

van Langevelde, H. J., Frail, D. A., Cordes, J. M., \& Diamond, P. J. 1992, ApJ, 396,686

Yusef-Zadeh, F., Cotton, W., et al. 1994, ApJ, 434, L63 